\title{
OHNO-TYPE IDENTITIES FOR MULTIPLE HARMONIC SUMS
}

\author{
SHIN-ICHIRO SEKI AND SHUJI YAMAMOTO
}

\begin{abstract}
We establish Ohno-type identities for multiple harmonic $(q-)$ sums which generalize Hoffman's identity and Bradley's identity. Our result leads to a new proof of the Ohno-type relation for $\mathcal{A}$-finite multiple zeta values recently proved by Hirose, Imatomi, Murahara and Saito. As a further application, we give certain sum formulas for $\mathcal{A}_{2}$ - or $\mathcal{A}_{3}$-finite multiple zeta values.
\end{abstract}

\section{INTRODUCTION}

Let $N$ be a positive integer. Euler [5] proved the following identity for the $N$-th harmonic number:

$$
\sum_{m=1}^{N} \frac{(-1)^{m-1}}{m}\left(\begin{array}{l}
N \\
m
\end{array}\right)=\sum_{n=1}^{N} \frac{1}{n} .
$$

It is known today that there are various generalizations of Euler's identity. We call a tuple of positive integers an index. For an index $\boldsymbol{k}=\left(k_{1}, \ldots, k_{r}\right)$, we write it in the form

$$
\boldsymbol{k}=\left(\{1\}^{a_{1}-1}, b_{1}+1, \ldots,\{1\}^{a_{s-1}-1}, b_{s-1}+1,\{1\}^{a_{s}-1}, b_{s}\right),
$$

where $a_{1}, \ldots, a_{s}, b_{1}, \ldots, b_{s}$ are positive integers and $\{1\}^{a}$ means $1, \ldots, 1$ repeated $a$ times, and then we define its Hoffman dual $\boldsymbol{k}^{\vee}$ by

$$
\boldsymbol{k}^{\vee}:=\left(a_{1},\{1\}^{b_{1}-1}, a_{2}+1,\{1\}^{b_{2}-1}, \ldots, a_{s}+1,\{1\}^{b_{s}-1}\right) .
$$

Let $\boldsymbol{k}=\left(k_{1}, \ldots, k_{r}\right)$ and $\boldsymbol{k}^{\vee}=\left(l_{1}, \ldots, l_{s}\right)$. After Roman [12] (the case $r=1$ ) and Hernandez [1] (the case $s=1$ ), Hoffman [8] proved

$$
\sum_{1 \leq m_{1} \leq \cdots \leq m_{r} \leq N} \frac{(-1)^{m_{r}-1}}{m_{1}^{k_{1}} \cdots m_{r}^{k_{r}}}\left(\begin{array}{c}
N \\
m_{r}
\end{array}\right)=\sum_{1 \leq n_{1} \leq \cdots \leq n_{s} \leq N} \frac{1}{n_{1}^{l_{1}} \cdots n_{s}^{l_{s}}} .
$$

There are also $q$-analogs of these identities. Let $q$ be a real number satisfying $0<$ $q<1$. For an integer $m$, we define the $q$-integer $[m]_{q}:=\frac{1-q^{m}}{1-q}$. When $0 \leq m \leq N$, we define the $q$-factorial $[m]_{q} !:=\prod_{a=1}^{m}[a]_{q}\left([0]_{q}:=1\right)$ and the $q$-binomial coefficient

2010 Mathematics Subject Classification. 11M32, 11B65.

Key words and phrases. Multiple harmonic sums, Ohno-type identities, Finite multiple zeta values, Sum formulas.

This work was supported in part by JSPS KAKENHI Grant Numbers JP18J00151, JP16H06336, JP16K13742, JP18K03221, as well as the KiPAS program 2013-2018 of the Faculty of Science and Technology at Keio University. 
$\left(\begin{array}{l}N \\ m\end{array}\right)_{q}:=\frac{[N]_{q} !}{[m]_{q} ![N-m]_{q} !}$. Van Hamme [19] proved a $q$-analog of Euler's identity (1)

$$
\sum_{m=1}^{N} \frac{(-1)^{m-1} q^{\frac{m(m+1)}{2}}}{[m]_{q}}\left(\begin{array}{l}
N \\
m
\end{array}\right)_{q}=\sum_{n=1}^{N} \frac{q^{n}}{[n]_{q}}
$$

After Dilcher [4] (the case $r=1$ ) and Prodinger [11] (the case $s=1$ ), Bradley [3] proved a $q$-analog of Hoffman's identity (2)

$$
\begin{aligned}
\sum_{1 \leq m_{1} \leq \cdots \leq m_{r} \leq N} \frac{q^{\left(k_{1}-1\right) m_{1}+\cdots+\left(k_{r}-1\right) m_{r}}}{\left[m_{1}\right]_{q}^{k_{1}} \cdots\left[m_{r}\right]_{q}^{k_{r}}} & \cdot(-1)^{m_{r}-1} q^{\frac{m_{r}\left(m_{r}+1\right)}{2}}\left(\begin{array}{c}
N \\
m_{r}
\end{array}\right)_{q} \\
& =\sum_{1 \leq n_{1} \leq \cdots \leq n_{s} \leq N} \frac{q^{n_{1}+\cdots+n_{s}}}{\left[n_{1}\right]_{q}^{l_{1}} \cdots\left[n_{s}\right]_{q}^{l_{s}}}
\end{aligned}
$$

The equality (2) or (3) is a kind of duality for multiple harmonic ( $q$-)sums. Since the duality relations for $(q-)$ multiple zeta values are generalized to Ohno's relations $([9,2])$, it is natural to ask whether (and how) we can generalize (2) and (3) to Ohno-type identities. This question was considered by Oyama [10] and more recently by Hirose, Imatomi, Murahara and Saito [7]. More precisely, they treated identities of the $\mathcal{A}$-finite multiple zeta values, that is, congruences modulo prime numbers.

In this article, we prove Ohno-type identities which generalize (3) (Theorem 2.1) and (2) (Corollary 2.2). We stress that our formulas are true identities, not congruences. This allows us to give, besides a new proof of Hirose-Imatomi-Murahara-Saito's relation for $\mathcal{A}$-finite multiple zeta values, sum formulas for $\mathcal{A}_{2^{-}}$or $\mathcal{A}_{3}$-finite multiple zeta values, which are congruences modulo square or cube of primes.

\section{Main Results}

2.1. Ohno-type identity. For a tuple of non-negative integers $\boldsymbol{e}=\left(e_{1}, \ldots, e_{r}\right)$, we define its weight wt $(\boldsymbol{e})$ and $\operatorname{depth} \operatorname{dep}(\boldsymbol{e})$ to be $e_{1}+\cdots+e_{r}$ and $r$, respectively. Let $J_{e, r}$ be the set of all tuples of non-negative integers $\boldsymbol{e} \operatorname{such}$ that $\operatorname{wt}(\boldsymbol{e})=e, \operatorname{dep}(\boldsymbol{e})=r$, and set $J_{*, r}:=\bigcup_{e=0}^{\infty} J_{e, r}$. For $\boldsymbol{e}_{1}, \boldsymbol{e}_{2} \in J_{*, r}, \boldsymbol{e}_{1}+\boldsymbol{e}_{2}$ denotes the entrywise sum. Similarly, let $I_{k, r}$ be the set of all indices $\boldsymbol{k}$ such that $\operatorname{wt}(\boldsymbol{k})=k, \operatorname{dep}(\boldsymbol{k})=r$, and set $I_{*, r}:=\bigcup_{k=0}^{\infty} I_{k, r}$. By convention, $I_{*, 0}=\{\varnothing\}$ is the set consisting only of the empty index.

For $\boldsymbol{k}=\left(k_{1}, \ldots, k_{r}\right) \in I_{*, r}$ and $\boldsymbol{e}=\left(e_{1}, \ldots, e_{r}\right) \in J_{*, r}$, put

$$
b(\boldsymbol{k} ; \boldsymbol{e}):=\prod_{i=1}^{r}\left(\begin{array}{c}
k_{i}+e_{i}+\delta_{i 1}+\delta_{i r}-2 \\
e_{i}
\end{array}\right),
$$

where $\delta_{i j}$ is Kronecker's delta. Here, we use the convention that

$$
\left(\begin{array}{c}
e-1 \\
e
\end{array}\right)= \begin{cases}1 & (e=0) \\
0 & (e>0)\end{cases}
$$


For a positive integer $N, \boldsymbol{k}=\left(k_{1}, \ldots, k_{r}\right) \in I_{*, r}$ and $\boldsymbol{e}=\left(e_{1}, \ldots, e_{r}\right) \in J_{*, r}$, we define the multiple harmonic $q$-sums $H_{N}^{\star}(\boldsymbol{k} ; q)$ and $z_{N}^{\star}(\boldsymbol{k} ; \boldsymbol{e} ; q)$ by

$$
\begin{aligned}
H_{N}^{\star}(\boldsymbol{k} ; q) & :=\sum_{1 \leq m_{1} \leq \cdots \leq m_{r} \leq N} \frac{q^{\left(k_{1}-1\right) m_{1}+\cdots+\left(k_{r}-1\right) m_{r}}}{\left[m_{1}\right]_{q}^{k_{1}} \cdots\left[m_{r}\right]_{q}^{k_{r}}} \cdot(-1)^{m_{r}-1} q^{\frac{m_{r}\left(m_{r}+1\right)}{2}}\left(\begin{array}{c}
N \\
m_{r}
\end{array}\right)_{q}, \\
z_{N}^{\star}(\boldsymbol{k} ; \boldsymbol{e} ; q) & :=\sum_{1 \leq m_{1} \leq \cdots \leq m_{r} \leq N} \frac{q^{\left(e_{1}+1\right) m_{1}+\cdots+\left(e_{r}+1\right) m_{r}}}{\left[m_{1}\right]_{q}^{k_{1}+e_{1}} \cdots\left[m_{r}\right]_{q}^{k_{r}+e_{r}}} .
\end{aligned}
$$

We set $z_{N}^{\star}(\boldsymbol{k} ; q):=z_{N}^{\star}\left(\boldsymbol{k} ;\{0\}^{r} ; q\right)$ and $z_{N}^{\star}(\varnothing ; q):=1$. The first main result is the following:

Theorem 2.1. Let $N$ be a positive integer, e a non-negative integer and $\boldsymbol{k} \in I_{*, r}$ an index. Set $s:=\operatorname{dep}\left(\boldsymbol{k}^{\vee}\right)$. Then we have

$$
\sum_{\boldsymbol{e} \in J_{e, r}} b(\boldsymbol{k} ; \boldsymbol{e}) H_{N}^{\star}(\boldsymbol{k}+\boldsymbol{e} ; q)=\sum_{j=0}^{e} z_{N}^{\star}\left(\{1\}^{e-j} ; q\right) \sum_{\boldsymbol{e}^{\prime} \in J_{j, s}} z_{N}^{\star}\left(\boldsymbol{k}^{\vee} ; \boldsymbol{e}^{\prime} ; q\right) \text {. }
$$

The case $e=0$ gives Bradley's identity $H_{N}^{\star}(\boldsymbol{k} ; q)=z_{N}^{\star}\left(\boldsymbol{k}^{\vee} ; q\right)$. We will prove (4) by using a certain connected sum in $₫ 3$, based on the same idea used in another paper of the authors [17]. This proof is new even if one specializes it to Hoffman's identity.

Let

$$
\begin{aligned}
H_{N}^{\star}(\boldsymbol{k}) & :=\lim _{q \rightarrow 1} H_{N}^{\star}(\boldsymbol{k} ; q)=\sum_{1 \leq m_{1} \leq \cdots \leq m_{r} \leq N} \frac{(-1)^{m_{r}-1}}{m_{1}^{k_{1}} \cdots m_{r}^{k_{r}}}\left(\begin{array}{c}
N \\
m_{r}
\end{array}\right), \\
\zeta_{N}^{\star}(\boldsymbol{k}): & :=\lim _{q \rightarrow 1} z_{N}^{\star}(\boldsymbol{k} ; q)=\sum_{1 \leq m_{1} \leq \cdots \leq m_{r} \leq N} \frac{1}{m_{1}^{k_{1}} \cdots m_{r}^{k_{r}}} .
\end{aligned}
$$

By taking the limit $q \rightarrow 1$ in (4), we obtain the following:

Corollary 2.2. Let $N$ be a positive integer, e a non-negative integer and $\boldsymbol{k} \in I_{*, r}$ an index. Set $s:=\operatorname{dep}\left(\boldsymbol{k}^{\vee}\right)$. Then we have

$$
\sum_{\boldsymbol{e} \in J_{e, r}} b(\boldsymbol{k} ; \boldsymbol{e}) H_{N}^{\star}(\boldsymbol{k}+\boldsymbol{e})=\sum_{j=0}^{e} \zeta_{N}^{\star}\left(\{1\}^{e-j}\right) \sum_{\boldsymbol{e}^{\prime} \in J_{j, s}} \zeta_{N}^{\star}\left(\boldsymbol{k}^{\vee}+\boldsymbol{e}^{\prime}\right) .
$$

The case $e=0$ gives Hoffman's identity $H_{N}^{\star}(\boldsymbol{k})=\zeta_{N}^{\star}\left(\boldsymbol{k}^{\vee}\right)$.

For an application of (6), we recall $\mathcal{A}$-finite multiple zeta values. First we define a $\mathbb{Q}$-algebra $\mathcal{A}$ by

$$
\mathcal{A}:=\left(\prod_{p: \text { prime }} \mathbb{Z} / p \mathbb{Z}\right) /\left(\bigoplus_{p: \text { prime }} \mathbb{Z} / p \mathbb{Z}\right) .
$$

For a positive integer $N$ and an index $\boldsymbol{k}=\left(k_{1}, \ldots, k_{r}\right) \in I_{*, r}$, we define a multiple harmonic sum $\zeta_{N}(\boldsymbol{k})$ by

$$
\zeta_{N}(\boldsymbol{k}):=\sum_{1 \leq m_{1}<\cdots<m_{r} \leq N} \frac{1}{m_{1}^{k_{1}} \cdots m_{r}^{k_{r}}}
$$


(compare with $\zeta_{N}^{\star}(\boldsymbol{k})$ given in (5) $)$. We set $\zeta_{N}(\varnothing)=\zeta_{N}^{\star}(\varnothing)=1$ by convention. Then the $\mathcal{A}$-finite multiple zeta values $\zeta_{\mathcal{A}}(\boldsymbol{k})$ and $\zeta_{\mathcal{A}}^{\star}(\boldsymbol{k})$ are defined by

$$
\zeta_{\mathcal{A}}(\boldsymbol{k}):=\left(\zeta_{p-1}(\boldsymbol{k}) \bmod p\right)_{p}, \quad \zeta_{\mathcal{A}}^{\star}(\boldsymbol{k}):=\left(\zeta_{p-1}^{\star}(\boldsymbol{k}) \bmod p\right)_{p} \in \mathcal{A} .
$$

Since $(-1)^{m-1}\left(\begin{array}{c}p-1 \\ m\end{array}\right) \equiv-1(\bmod p)$ holds for any prime $p$ greater than $m$, we have

$$
\left(H_{p-1}^{\star}(\boldsymbol{k}) \bmod p\right)_{p}=-\zeta_{\mathcal{A}}^{\star}(\boldsymbol{k}) \text {. }
$$

Moreover, it is known that $\zeta_{\mathcal{A}}^{\star}\left(\{1\}^{e}\right)=0$ for $e>0$, while $\zeta_{\mathcal{A}}^{\star}(\varnothing)=1$. Hence we obtain the following relation among $\mathcal{A}$-finite multiple zeta values as a corollary of (6) $)$.

Corollary 2.3 (Hirose-Imatomi-Murahara-Saito [7). Let e be a non-negative integer and $\boldsymbol{k} \in I_{*, r}$ an index. Set $s:=\operatorname{dep}\left(\boldsymbol{k}^{\vee}\right)$. Then we have

$$
\sum_{\boldsymbol{e} \in J_{e, r}} b(\boldsymbol{k} ; \boldsymbol{e}) \zeta_{\mathcal{A}}^{\star}(\boldsymbol{k}+\boldsymbol{e})=-\sum_{e^{\prime} \in J_{e, s}} \zeta_{\mathcal{A}}^{\star}\left(\boldsymbol{k}^{\vee}+\boldsymbol{e}^{\prime}\right)
$$

2.2. Sum formulas for finite multiple zeta values. Before stating our second main result, let us recall the sum formulas for $\mathcal{A}$-finite multiple zeta values. First, it is easily seen that

$$
\sum_{\boldsymbol{k} \in I_{k, r}} \zeta_{\mathcal{A}}(\boldsymbol{k})=\sum_{\boldsymbol{k} \in I_{k, r}} \zeta_{\mathcal{A}}^{\star}(\boldsymbol{k})=0
$$

but this is not an analog of the sum formula for the multiple zeta values [6], since the admissibility condition $k_{r} \geq 2$ is ignored in (7). A more precise analog (and its generalization) is due to Saito-Wakabayashi [14]. For integers $k, r$ and $i$ satisfying $1 \leq$ $i \leq r<k$, we put $I_{k, r, i}:=\left\{\left(k_{1}, \ldots, k_{r}\right) \in I_{k, r} \mid k_{i} \geq 2\right\}$ and $B_{p-k}:=\left(B_{p-k} \bmod p\right)_{p} \in \mathcal{A}$, where $B_{n}$ denotes the $n$-th Seki-Bernoulli number. Note that $B_{\boldsymbol{p}-k}=0$ if $k$ is even.

Theorem 2.4 (Saito-Wakabayashi [14]). Let $k, r$ and $i$ be integers satisfying $1 \leq i \leq$ $r<k$. Then, in the ring $\mathcal{A}$, we have equalities

$$
\begin{aligned}
\sum_{\boldsymbol{k} \in I_{k, r, i}} \zeta_{\mathcal{A}}(\boldsymbol{k}) & =(-1)^{i}\left\{\left(\begin{array}{c}
k-1 \\
i-1
\end{array}\right)+(-1)^{r}\left(\begin{array}{c}
k-1 \\
r-i
\end{array}\right)\right\} \frac{B_{\boldsymbol{p}-k}}{k}, \\
\sum_{\boldsymbol{k} \in I_{k, r, i}} \zeta_{\mathcal{A}}^{\star}(\boldsymbol{k}) & =(-1)^{i}\left\{\left(\begin{array}{c}
k-1 \\
r-i
\end{array}\right)+(-1)^{r}\left(\begin{array}{c}
k-1 \\
i-1
\end{array}\right)\right\} \frac{B_{\boldsymbol{p}-k}}{k} .
\end{aligned}
$$

In particular, if $k$ is even, we see that

$$
\sum_{\boldsymbol{k} \in I_{k, r, i}} \zeta_{\mathcal{A}}(\boldsymbol{k})=\sum_{\boldsymbol{k} \in I_{k, r, i}} \zeta_{\mathcal{A}}^{\star}(\boldsymbol{k})=0 .
$$

Our aim is to lift the identities (17) and (8) in $\mathcal{A}$, which represent systems of congruences modulo (almost all) primes $p$, to congruences modulo $p^{2}$ or $p^{3}$, by using the identity (6) .

Let $n$ be a positive integer. In accordance with [13, 16, 21], we define a $\mathbb{Q}$-algebra $\mathcal{A}_{n}$ by

$$
\mathcal{A}_{n}:=\left(\prod_{p: \text { prime }} \mathbb{Z} / p^{n} \mathbb{Z}\right) /\left(\bigoplus_{p: \text { prime }} \mathbb{Z} / p^{n} \mathbb{Z}\right)
$$


and the $\mathcal{A}_{n}$-finite multiple zeta values $\zeta_{\mathcal{A}_{n}}(\boldsymbol{k})$ and $\zeta_{\mathcal{A}_{n}}^{\star}(\boldsymbol{k})$ by

$$
\zeta_{\mathcal{A}_{n}}(\boldsymbol{k}):=\left(\zeta_{p-1}(\boldsymbol{k}) \bmod p^{n}\right)_{p}, \quad \zeta_{\mathcal{A}_{n}}^{\star}(\boldsymbol{k}):=\left(\zeta_{p-1}^{\star}(\boldsymbol{k}) \bmod p^{n}\right)_{p} \in \mathcal{A}_{n} .
$$

We use the symbol $B_{p-k}$ again to denote the element $\left(B_{p-k} \bmod p^{n}\right)_{p}$ of $\mathcal{A}_{n}$, and put $\boldsymbol{p}:=\left(p \bmod p^{n}\right)_{p} \in \mathcal{A}_{n}$. Then our second main result is the following:

Theorem 2.5 (= Proposition 4.6 + Theorem 5.1 + Theorem 4.7). Let $k, r$ be positive integers satisfying $r \leq k$. Then, in the ring $\mathcal{A}_{2}$, we have

$$
\sum_{\boldsymbol{k} \in I_{k, r}} \zeta_{\mathcal{A}_{2}}(\boldsymbol{k})=(-1)^{r-1}\left(\begin{array}{l}
k \\
r
\end{array}\right) \frac{B_{\boldsymbol{p}-k-1}}{k+1} \boldsymbol{p}, \quad \sum_{\boldsymbol{k} \in I_{k, r}} \zeta_{\mathcal{A}_{2}}^{\star}(\boldsymbol{k})=\left(\begin{array}{l}
k \\
r
\end{array}\right) \frac{B_{\boldsymbol{p}-k-1}}{k+1} \boldsymbol{p} .
$$

If $k$ is odd, in the ring $\mathcal{A}_{3}$, we have

$$
\sum_{\boldsymbol{k} \in I_{k, r}} \zeta_{\mathcal{A}_{3}}(\boldsymbol{k})=(-1)^{r} \frac{k+1}{2}\left(\begin{array}{l}
k \\
r
\end{array}\right) \frac{B_{\boldsymbol{p}-k-2}}{k+2} \boldsymbol{p}^{2}, \quad \sum_{\boldsymbol{k} \in I_{k, r}} \zeta_{\mathcal{A}_{3}}^{\star}(\boldsymbol{k})=-\frac{k+1}{2}\left(\begin{array}{l}
k \\
r
\end{array}\right) \frac{B_{\boldsymbol{p}-k-2}}{k+2} \boldsymbol{p}^{2} .
$$

Furthermore, let $i$ be an integer satisfying $1 \leq i \leq r$ and we assume that $k$ is even and greater than $r$. Then the equalities

$$
\sum_{\boldsymbol{k} \in I_{k, r, i}} \zeta_{\mathcal{A}_{2}}(\boldsymbol{k})=(-1)^{r-1} \frac{a_{k, r, i}}{2} \cdot \frac{B_{\boldsymbol{p}-k-1}}{k+1} \boldsymbol{p}, \quad \sum_{\boldsymbol{k} \in I_{k, r, i}} \zeta_{\mathcal{A}_{2}}^{\star}(\boldsymbol{k})=\frac{b_{k, r, i}}{2} \cdot \frac{B_{\boldsymbol{p}-k-1}}{k+1} \boldsymbol{p}
$$

hold in $\mathcal{A}_{2}$. Here the coefficients $a_{k, r, i}$ and $b_{k, r, i}$ are given by

$$
\begin{aligned}
a_{k, r, i} & :=\left(\begin{array}{c}
k-1 \\
r
\end{array}\right)+(-1)^{r-i}\left\{(k-r)\left(\begin{array}{c}
k \\
i-1
\end{array}\right)+\left(\begin{array}{c}
k-1 \\
i-1
\end{array}\right)+(-1)^{r-1}\left(\begin{array}{c}
k-1 \\
r-i
\end{array}\right)\right\}, \\
b_{k, r, i} & :=\left(\begin{array}{c}
k-1 \\
r
\end{array}\right)+(-1)^{i-1}\left\{(k-r)\left(\begin{array}{c}
k \\
r-i
\end{array}\right)+\left(\begin{array}{c}
k-1 \\
r-i
\end{array}\right)+(-1)^{r-1}\left(\begin{array}{c}
k-1 \\
i-1
\end{array}\right)\right\} .
\end{aligned}
$$

We will prove this theorem in 94 and $\$ 5$,

\section{The Proof of TheOrem 2.1}

Definition 3.1 (Connected sum). Let $N$ be a positive integer, $q$ a real number satisfying $0<q<1$ and $x$ an indeterminate. Let $r>0$ and $s \geq 0$ be integers. For $\boldsymbol{k}=$ $\left(k_{1}, \ldots, k_{r}\right) \in J_{*, r}$ satisfying $k_{1}, \ldots, k_{r-1} \geq 1$ and $\boldsymbol{l}=\left(l_{1}, \ldots, l_{s}\right) \in I_{*, s}$, we define a formal power series $Z_{N}^{\star}(\boldsymbol{k} ; \boldsymbol{l} ; q ; x)$ in $x$ by

$$
Z_{N}^{\star}(\boldsymbol{k} ; \boldsymbol{l} ; q ; x):=\sum_{1 \leq m_{1} \leq \cdots \leq m_{r} \leq n_{1} \leq \cdots \leq n_{s} \leq n_{s+1}=N} F_{1}(\boldsymbol{k} ; \boldsymbol{m} ; q ; x) C\left(m_{r}, n_{1}, q, x\right) F_{2}(\boldsymbol{l} ; \boldsymbol{n} ; q ; x),
$$

where

$$
\begin{aligned}
F_{1}(\boldsymbol{k} ; \boldsymbol{m} ; q ; x) & :=\frac{\left[m_{1}\right]_{q}}{\left[m_{1}\right]_{q}-q^{m_{1}} x} \prod_{i=1}^{r} \frac{q^{\left(k_{i}-1\right) m_{i}}}{\left[m_{i}\right]_{q}\left(\left[m_{i}\right]_{q}-q^{m_{i}} x\right)^{k_{i}-1}} \cdot \frac{\left[m_{r}\right]_{q}}{\left[m_{r}\right]_{q}-q^{m_{r}} x} \\
C\left(m_{r}, n_{1}, q, x\right) & :=(-1)^{m_{r}-1} q^{\frac{m_{r}\left(m_{r}+1\right)}{2}} \frac{\prod_{h=1}^{n_{1}}\left([h]_{q}-q^{h} x\right)}{\left[m_{r}\right]_{q} !\left[n_{1}-m_{r}\right]_{q} !} \\
F_{2}(\boldsymbol{l} ; \boldsymbol{n} ; q ; x) & :=\prod_{j=1}^{s} \frac{q^{n_{j}}}{\left(\left[n_{j}\right]_{q}-q^{n_{j}} x\right)\left[n_{j}\right]_{q}^{l_{j}-1}}
\end{aligned}
$$


for $\boldsymbol{m}=\left(m_{1}, \ldots, m_{r}\right)$ and $\boldsymbol{n}=\left(n_{1}, \ldots, n_{s}\right)$.

Remark 3.2. The sum $Z_{N}^{\star}(\boldsymbol{k} ; \boldsymbol{l} ; q ; x)$ consists of two parts

$$
\sum_{1 \leq m_{1} \leq \cdots \leq m_{r} \leq N} F_{1}(\boldsymbol{k} ; \boldsymbol{m} ; q ; x) \text { and } \sum_{1 \leq n_{1} \leq \cdots \leq n_{s} \leq N} F_{2}(\boldsymbol{l} ; \boldsymbol{n} ; q ; x)
$$

connected by the factor $C\left(m_{r}, n_{1}, q, x\right)$ (and the relation $\left.m_{r} \leq n_{1}\right)$. We call it a connected sum with connector $C\left(m_{r}, n_{1}, q, x\right)$. In [17], another type of connected sums is used to give a new proof of Ohno's relation for the multiple zeta values and Bradley's $q$-analog of it.

Theorem 3.3. For $\left(k_{1}, \ldots, k_{r}\right) \in J_{*, r}$ with $k_{1}, \ldots, k_{r-1} \geq 1$ and $\left(l_{1}, \ldots, l_{s}\right) \in I_{*, s}$, we have

$$
Z_{N}^{\star}\left(k_{1}, \ldots, k_{r}+1 ; l_{1}, \ldots, l_{s} ; q ; x\right)=Z_{N}^{\star}\left(k_{1}, \ldots, k_{r} ; 1, l_{1}, \ldots, l_{s} ; q ; x\right) .
$$

Moreover, if $s>0$, we also have

$$
Z_{N}^{\star}\left(k_{1}, \ldots, k_{r}+1,0 ; l_{1}, \ldots, l_{s} ; q ; x\right)=Z_{N}^{\star}\left(k_{1}, \ldots, k_{r} ; 1+l_{1}, \ldots, l_{s} ; q ; x\right) .
$$

Proof. The equality (9) follows from the telescoping sum

$$
\begin{aligned}
& \frac{q^{m}}{[m]_{q}-q^{m} x} \cdot C(m, n, q, x) \\
& =\sum_{a=m}^{n}\left(\frac{q^{m}}{[m]_{q}-q^{m} x} \cdot C(m, a, q, x)-\frac{q^{m}}{[m]_{q}-q^{m} x} \cdot C(m, a-1, q, x)\right) \\
& =\sum_{a=m}^{n} C(m, a, q, x) \cdot \frac{q^{a}}{[a]_{q}-q^{a} x}
\end{aligned}
$$

applied to $m=m_{r}, n=n_{2}$ and $a=n_{1}$ in the definition of $Z_{N}^{\star}\left(k_{1}, \ldots, k_{r} ; 1, l_{1}, \ldots, l_{s} ; q ; x\right)$. Similarly, the equality (10) follows from the telescoping sum

$$
\begin{aligned}
& \frac{q^{m}}{[m]_{q}} \sum_{a=m}^{n} q^{-a} C(a, n, q, x) \\
& =\frac{q^{m}}{[m]_{q}} \sum_{a=m}^{n}\left(\frac{[a]_{q}}{q^{a}} \cdot C(a, n, q, x) \cdot \frac{1}{[n]_{q}}-\frac{[a+1]_{q}}{q^{a+1}} \cdot C(a+1, n, q, x) \cdot \frac{1}{[n]_{q}}\right) \\
& =C(m, n, q, x) \cdot \frac{1}{[n]_{q}}
\end{aligned}
$$

applied to $m=m_{r}, n=n_{1}, a=m_{r+1}$ in the definition of $Z_{N}^{\star}\left(k_{1}, \ldots, k_{r}+1,0 ; l_{1}, \ldots, l_{s} ; q ; x\right)$. 
Corollary 3.4. Let $N$ be a positive integer and $\boldsymbol{k}=\left(k_{1}, \ldots, k_{r}\right)$ an index. We define $P_{N}(\boldsymbol{k} ; q ; x), Q_{N}(\boldsymbol{k} ; q ; x)$ and $R_{N}(q ; x)$ by

$$
\begin{aligned}
& P_{N}(\boldsymbol{k} ; q ; x):=\sum_{1 \leq m_{1} \leq \cdots \leq m_{r} \leq N} \frac{\left[m_{1}\right]_{q}}{\left[m_{1}\right]_{q}-q^{m_{1}} x} \prod_{i=1}^{r} \frac{q^{\left(k_{i}-1\right) m_{i}}}{\left[m_{i}\right]_{q}\left(\left[m_{i}\right]_{q}-q^{m_{i}} x\right)^{k_{i}-1}} \cdot \frac{\left[m_{r}\right]_{q}}{\left[m_{r}\right]_{q}-q^{m_{r}} x} \\
& \cdot(-1)^{m_{r}-1} q^{\frac{m_{r}\left(m_{r}+1\right)}{2}}\left(\begin{array}{c}
N \\
m_{r}
\end{array}\right)_{q}, \\
& Q_{N}(\boldsymbol{k} ; q ; x):=\sum_{1 \leq m_{1} \leq \cdots \leq m_{r} \leq N} \prod_{i=1}^{r} \frac{q^{m_{i}}}{\left(\left[m_{i}\right]_{q}-q^{m_{i}} x\right)\left[m_{i}\right]_{q}^{k_{i}-1}}, \\
& R_{N}(q ; x):=\prod_{h=1}^{N}\left(1-\frac{q^{h} x}{[h]_{q}}\right)^{-1} .
\end{aligned}
$$

Then we have

$$
P_{N}(\boldsymbol{k} ; q ; x)=Q_{N}\left(\boldsymbol{k}^{\vee} ; q ; x\right) R_{N}(q ; x) .
$$

Proof. By applying equalities in Theorem $3.3 \mathrm{wt}(\boldsymbol{k})$ times, we see that

$$
Z_{N}^{\star}(\boldsymbol{k} ; \varnothing ; q ; x)=\cdots=Z_{N}^{\star}\left(0 ; \boldsymbol{k}^{\vee} ; q ; x\right)
$$

holds by the definition of the Hoffman dual. For example,

$$
Z_{N}^{\star}(1,1,2 ; \varnothing) \stackrel{(\underline{\underline{9}})}{=} Z_{N}^{\star}(1,1,1 ; 1) \stackrel{(9)}{=} Z_{N}^{\star}(1,1,0 ; 1,1) \stackrel{(10)}{=} Z_{N}^{\star}(1,0 ; 2,1) \stackrel{(10)}{=} Z_{N}^{\star}(0 ; 3,1)
$$

(here we abbreviated $Z_{N}^{\star}(\boldsymbol{k} ; \boldsymbol{l} ; q ; x)$ as $\left.Z_{N}^{\star}(\boldsymbol{k} ; \boldsymbol{l})\right)$. By definition, we have

$$
\begin{aligned}
Z_{N}^{\star}(\boldsymbol{k} ; \varnothing ; q ; x) & =\sum_{1 \leq m_{1} \leq \cdots \leq m_{r} \leq N} F_{1}(\boldsymbol{k} ; \boldsymbol{m} ; q ; x) C\left(m_{r}, N, q, x\right) \\
& =P_{N}(\boldsymbol{k} ; q ; x) R_{N}(q ; x)^{-1}
\end{aligned}
$$

and

$$
\begin{aligned}
Z_{N}^{\star}\left(0 ; \boldsymbol{k}^{\vee} ; q ; x\right) & =\sum_{\substack{1 \leq m \leq n_{1} \leq \cdots \leq n_{s} \leq N\\
}} \frac{q^{-m}[m]_{q}}{[m]_{q}-q^{m} x} C\left(m, n_{1}, q, x\right) F_{2}\left(\boldsymbol{k}^{\vee} ; \boldsymbol{n} ; q ; x\right) \\
& =Q_{N}\left(\boldsymbol{k}^{\vee} ; q ; x\right) .
\end{aligned}
$$

In the last equality, we have used the partial fraction decomposition

$$
\sum_{m=1}^{n_{1}} \frac{[m]_{q}}{[m]_{q}-q^{m} x} \cdot \frac{(-1)^{m-1} q^{\frac{m(m-1)}{2}}}{[m]_{q} !\left[n_{1}-m\right]_{q} !}=\frac{1}{\prod_{h=1}^{n_{1}}\left([h]_{q}-q^{h} x\right)}
$$

The proof is complete.

Proof of Theorem 2.1. By using the expansion formula

$$
\frac{1}{\left([m]_{q}-q^{m} x\right)^{k}}=\sum_{e=0}^{\infty}\left(\begin{array}{c}
k+e-1 \\
e
\end{array}\right) \frac{q^{e m} x^{e}}{[m]_{q}^{k+e}}
$$


for a positive integer $m$ and a non-negative integer $k$, we see that

$$
P_{N}(\boldsymbol{k} ; q ; x)=\sum_{e=0}^{\infty} \sum_{\boldsymbol{e} \in J_{e, r}} b(\boldsymbol{k} ; \boldsymbol{e}) H_{N}^{\star}(\boldsymbol{k}+\boldsymbol{e} ; q) x^{e}
$$

and

$$
Q_{N}\left(\boldsymbol{k}^{\vee} ; q ; x\right)=\sum_{e=0}^{\infty} \sum_{\boldsymbol{e} \in J_{e, s}} z_{N}^{\star}\left(\boldsymbol{k}^{\vee} ; \boldsymbol{e} ; q\right) x^{e} .
$$

Since $R_{N}(q ; x)=\sum_{e=0}^{\infty} z_{N}^{\star}\left(\{1\}^{e} ; q\right) x^{e}$, we obtain the identity (4) by comparing the coefficients of $x^{e}$ in (11).

\section{Sum formulas fOR $\mathcal{A}_{2}$-Finite multiple Zeta VAlues}

4.1. Auxiliary facts. We prepare some known facts for finite multiple zeta values.

Proposition 4.1 ([8, Theorem 6.1, 6.2], [20, Theorem 3.1, 3.5]). Let $k_{1}, k_{2}$ and $k_{3}$ be positive integers, and assume that $l:=k_{1}+k_{2}+k_{3}$ is odd. Then

$$
\begin{aligned}
\zeta_{\mathcal{A}}^{\star}\left(k_{1}, k_{2}\right) & =(-1)^{k_{2}}\left(\begin{array}{c}
k_{1}+k_{2} \\
k_{1}
\end{array}\right) \frac{B_{\boldsymbol{p}-k_{1}-k_{2}}}{k_{1}+k_{2}}, \\
\zeta_{\mathcal{A}}^{\star}\left(k_{1}, k_{2}, k_{3}\right) & =\frac{1}{2}\left\{(-1)^{k_{3}}\left(\begin{array}{c}
l \\
k_{3}
\end{array}\right)-(-1)^{k_{1}}\left(\begin{array}{c}
l \\
k_{1}
\end{array}\right)\right\} \frac{B_{\boldsymbol{p}-l}}{l} .
\end{aligned}
$$

Proposition 4.2 ([22], [20, Theorem 3.2]). Let $k, r, k_{1}$ and $k_{2}$ be positive integers, and assume that $l:=k_{1}+k_{2}$ is even. Then

$$
\begin{aligned}
\zeta_{\mathcal{A}_{2}}^{\star}\left(\{k\}^{r}\right) & =k \frac{B_{\boldsymbol{p}-r k-1}}{r k+1} \boldsymbol{p}, \\
\zeta_{\mathcal{A}_{2}}^{\star}\left(k_{1}, k_{2}\right) & =\frac{1}{2}\left\{(-1)^{k_{1}} k_{2}\left(\begin{array}{c}
l+1 \\
k_{1}
\end{array}\right)-(-1)^{k_{2}} k_{1}\left(\begin{array}{c}
l+1 \\
k_{2}
\end{array}\right)+l\right\} \frac{B_{\boldsymbol{p}-l-1}}{l+1} \boldsymbol{p} .
\end{aligned}
$$

Proposition 4.3 ([15, Corollary $3.16(42)])$. Let $n$ be a positive integer and $\boldsymbol{k}=$ $\left(k_{1}, \ldots, k_{r}\right)$ an index. Then

$$
\sum_{j=0}^{r}(-1)^{j} \zeta_{\mathcal{A}_{n}}\left(k_{j}, \ldots, k_{1}\right) \zeta_{\mathcal{A}_{n}}^{\star}\left(k_{j+1}, \ldots, k_{r}\right)=0 .
$$

\subsection{Computations of sums for $\mathcal{A}_{2}$-finite multiple zeta values.}

Definition 4.4. Let $k, r$ and $i$ be positive integers satisfying $i \leq r \leq k$. We define four sums $S_{k, r}, S_{k, r}^{\star}, S_{k, r, i}$ and $S_{k, r, i}^{\star}$ in $\mathcal{A}_{2}$ by

$$
\begin{aligned}
S_{k, r} & :=\sum_{\boldsymbol{k} \in I_{k, r}} \zeta_{\mathcal{A}_{2}}(\boldsymbol{k}), & S_{k, r}^{\star} & :=\sum_{\boldsymbol{k} \in I_{k, r}} \zeta_{\mathcal{A}_{2}}^{\star}(\boldsymbol{k}), \\
S_{k, r, i} & :=\sum_{\boldsymbol{k} \in I_{k, r, i}} \zeta_{\mathcal{A}_{2}}(\boldsymbol{k}), & S_{k, r, i}^{\star} & :=\sum_{\boldsymbol{k} \in I_{k, r, i}} \zeta_{\mathcal{A}_{2}}^{\star}(\boldsymbol{k}) .
\end{aligned}
$$

For an index $\boldsymbol{k}=\left(k_{1}, \ldots, k_{r}\right)$, we set $\boldsymbol{k}^{+}:=\left(k_{1}, \ldots, k_{r-1}, k_{r}+1\right)$. We can calculate $S_{k, r}^{\star}$ and $S_{k, r, i}^{\star}$ by using the following identity. 
Corollary 4.5. Let e be a non-negative integer, $\boldsymbol{k} \in I_{*, r}$ an index and $s:=\operatorname{dep}\left(\boldsymbol{k}^{\vee}\right)$. Then we have

$$
\begin{aligned}
& \sum_{j=0}^{e} \zeta_{\mathcal{A}_{2}}^{\star}\left(\{1\}^{e-j}\right) \sum_{\boldsymbol{e} \in J_{j, r}} \zeta_{\mathcal{A}_{2}}^{\star}(\boldsymbol{k}+\boldsymbol{e}) \\
& =\sum_{\boldsymbol{e}^{\prime} \in J_{e, s}} b\left(\boldsymbol{k}^{\vee} ; \boldsymbol{e}^{\prime}\right)\left\{-\zeta_{\mathcal{A}_{2}}^{\star}\left(\boldsymbol{k}^{\vee}+\boldsymbol{e}^{\prime}\right)-\zeta_{\mathcal{A}_{2}}^{\star}\left(\boldsymbol{k}^{\vee}+\boldsymbol{e}^{\prime}, 1\right) \boldsymbol{p}+\zeta_{\mathcal{A}_{2}}^{\star}\left(\left(\boldsymbol{k}^{\vee}+\boldsymbol{e}^{\prime}\right)^{+}\right) \boldsymbol{p}\right\} .
\end{aligned}
$$

Proof. Since a congruence

$$
(-1)^{m-1}\left(\begin{array}{c}
p-1 \\
m
\end{array}\right) \equiv-1-\sum_{m \leq n \leq p-1} \frac{p}{n}+\frac{p}{m} \quad\left(\bmod p^{2}\right)
$$

holds for any odd prime $p$ and any positive integer $m$ with $m<p$ (cf. [16, Lemma 4.1]), this corollary is a direct consequence of (6) $)$.

Proposition 4.6. For positive integers $k$ and $r$ such that $r \leq k$, we have

$$
(-1)^{r-1} S_{k, r}=S_{k, r}^{\star}=\left(\begin{array}{c}
k \\
r
\end{array}\right) \frac{B_{\boldsymbol{p}-k-1}}{k+1} \boldsymbol{p} .
$$

Proof. Let $\boldsymbol{k}=\left(\{1\}^{r}\right)$ and $e=k-r$ in (17). Then $\boldsymbol{k}^{\vee}=(r)$ and we have

$$
\sum_{j=0}^{k-r} \zeta_{\mathcal{A}_{2}}^{\star}\left(\{1\}^{k-r-j}\right) S_{j+r, r}^{\star}=\left(\begin{array}{l}
k \\
r
\end{array}\right)\left\{-\zeta_{\mathcal{A}_{2}}(k)-\zeta_{\mathcal{A}_{2}}^{\star}(k, 1) \boldsymbol{p}+\zeta_{\mathcal{A}_{2}}(k+1) \boldsymbol{p}\right\} .
$$

For $0 \leq j<k-r, \zeta_{\mathcal{A}_{2}}^{\star}\left(\{1\}^{k-r-j}\right) S_{j+r, r}^{\star}=0$ since both $\zeta_{\mathcal{A}_{2}}^{\star}\left(\{1\}^{k-r-j}\right)$ and $S_{j+r, r}^{\star}$ are divisible by $\boldsymbol{p}$ by (14) and (7). Therefore, the left hand side of (18) is equal to $S_{k, r}^{\star}$. On the other hand, the right hand side of (18) is equal to

$$
\left(\begin{array}{c}
k \\
r
\end{array}\right)\left\{-k \frac{B_{\boldsymbol{p}-k-1}}{k+1} \boldsymbol{p}+\left(\begin{array}{c}
k+1 \\
k
\end{array}\right) \frac{B_{\boldsymbol{p}-k-1}}{k+1} \boldsymbol{p}\right\}=\left(\begin{array}{c}
k \\
r
\end{array}\right) \frac{B_{\boldsymbol{p}-k-1}}{k+1} \boldsymbol{p}
$$

by (14) and (12). Hence we have $S_{k, r}^{\star}=\left(\begin{array}{l}k \\ r\end{array}\right) \frac{B_{\boldsymbol{p}-k-1}}{k+1} \boldsymbol{p}$.

By taking $\sum_{k \in I_{k, r}}$ of (16) $)$, we obtain

$$
S_{k, r}^{\star}+\sum_{j=1}^{r-1}(-1)^{j} \sum_{l=j}^{k-r+j} S_{l, j} S_{k-l, r-j}^{\star}+(-1)^{r} S_{k, r}=0 .
$$

We see that $S_{l, j} S_{k-l, r-j}^{\star}=0$ for $1 \leq j \leq r-1$ and $j \leq l \leq k-r+j$, since both $S_{l, j}$ and $S_{k-l, r-j}^{\star}$ are divisible by $\boldsymbol{p}$ by (7). This gives $(-1)^{r-1} S_{k, r}=S_{k, r}^{\star}$.

Next we compute $S_{k, r, i}^{\star}$ and $S_{k, r, i}$.

Theorem 4.7. Let $k, r$ and $i$ be positive integers satisfying $i \leq r<k$, and assume that $k$ is even. Then we have

$$
S_{k, r, i}=(-1)^{r-1} \frac{a_{k, r, i}}{2} \cdot \frac{B_{\boldsymbol{p}-k-1}}{k+1} \boldsymbol{p}, \quad S_{k, r, i}^{\star}=\frac{b_{k, r, i}}{2} \cdot \frac{B_{\boldsymbol{p}-k-1}}{k+1} \boldsymbol{p},
$$


where

$$
\begin{aligned}
& a_{k, r, i}=\left(\begin{array}{c}
k-1 \\
r
\end{array}\right)+(-1)^{r-i}\left\{(k-r)\left(\begin{array}{c}
k \\
i-1
\end{array}\right)+\left(\begin{array}{c}
k-1 \\
i-1
\end{array}\right)+(-1)^{r-1}\left(\begin{array}{c}
k-1 \\
r-i
\end{array}\right)\right\} \\
& b_{k, r, i}=\left(\begin{array}{c}
k-1 \\
r
\end{array}\right)+(-1)^{i-1}\left\{(k-r)\left(\begin{array}{c}
k \\
r-i
\end{array}\right)+\left(\begin{array}{c}
k-1 \\
r-i
\end{array}\right)+(-1)^{r-1}\left(\begin{array}{c}
k-1 \\
i-1
\end{array}\right)\right\} .
\end{aligned}
$$

Proof. Let $\boldsymbol{k}=\left(\{1\}^{i-1}, 2,\{1\}^{r-i}\right)$ and $e=k-r-1$ in (17). Then $\boldsymbol{k}^{\vee}=(i, r-i+1)$ and we have

$$
\begin{aligned}
& \sum_{j=0}^{k-r-1} \zeta_{\mathcal{A}_{2}}^{\star}\left(\{1\}^{k-r-1-j}\right) S_{j+r+1, r, i}^{\star} \\
& =\sum_{e=0}^{k-r-1}\left(\begin{array}{c}
i+e-1 \\
e
\end{array}\right)\left(\begin{array}{l}
k-i-e-1 \\
k-r-1-e
\end{array}\right)\left\{-\zeta_{\mathcal{A}_{2}}^{\star}(i+e, k-i-e)\right. \\
& \left.\quad-\zeta_{\mathcal{A}_{2}}^{\star}(i+e, k-i-e, 1) \boldsymbol{p}+\zeta_{\mathcal{A}_{2}}^{\star}(i+e, k-i-e+1) \boldsymbol{p}\right\} .
\end{aligned}
$$

For $0 \leq j<k-r-1$, we see that $\zeta_{\mathcal{A}_{2}}^{\star}\left(\{1\}^{k-r-1-j}\right) S_{j+r+1, r, i}^{\star}$ is a rational multiple of $B_{\boldsymbol{p}-k+r+j} B_{\boldsymbol{p}-j-r-1} \boldsymbol{p}$ by (14) and Theorem 2.4. Since $k$ is even, one of $B_{\boldsymbol{p}-k+r+j}$ or $B_{p-j-r-1}$ is zero. Therefore, the left hand side of (19) is equal to $S_{k, r, i}^{\star}$.

On the other hand, we can calculate the right hand side of (19) as follows. By (15), (12) and (13), we have

$$
\begin{aligned}
- & \zeta_{\mathcal{A}_{2}}^{\star}(i+e, k-i-e)-\zeta_{\mathcal{A}_{2}}^{\star}(i+e, k-i-e, 1) \boldsymbol{p}+\zeta_{\mathcal{A}_{2}}^{\star}(i+e, k-i-e+1) \boldsymbol{p} \\
= & {\left[-\frac{1}{2}\left\{(-1)^{i+e}(k-i-e)\left(\begin{array}{c}
k+1 \\
i+e
\end{array}\right)-(-1)^{k-i-e}(i+e)\left(\begin{array}{c}
k+1 \\
k-i-e
\end{array}\right)+k\right\}\right.} \\
& \left.-\frac{1}{2}\left\{-(k+1)-(-1)^{i+e}\left(\begin{array}{c}
k+1 \\
i+e
\end{array}\right)\right\}+(-1)^{k-i-e+1}\left(\begin{array}{c}
k+1 \\
i+e
\end{array}\right)\right] \frac{B_{\boldsymbol{p}-k-1}}{k+1} \boldsymbol{p} \\
= & \frac{1}{2}\left[1-(-1)^{i+e}(k-i-e+1)\left(\begin{array}{c}
k+1 \\
i+e
\end{array}\right)+(-1)^{i+e}(i+e)\left(\begin{array}{c}
k+1 \\
k-i-e
\end{array}\right)\right] \frac{B_{\boldsymbol{p}-k-1}}{k+1} \boldsymbol{p} \\
= & \frac{1}{2}\left[1+(-1)^{i-1+e}\left(\begin{array}{c}
k+1 \\
i+e+1
\end{array}\right)\right] \frac{B_{\boldsymbol{p}-k-1}}{k+1} \boldsymbol{p} .
\end{aligned}
$$

Therefore, the right hand side of (19) is equal to

$$
\frac{1}{2} \sum_{e=0}^{k-r-1}\left(\begin{array}{c}
i+e-1 \\
e
\end{array}\right)\left(\begin{array}{c}
k-i-e-1 \\
k-r-1-e
\end{array}\right)\left[1+(-1)^{i-1+e}\left(\begin{array}{c}
k+1 \\
i+e+1
\end{array}\right)\right] \frac{B_{\boldsymbol{p}-k-1}}{k+1} \boldsymbol{p} .
$$

By comparing the coefficient of $x^{k-r-1}$ in $(1-x)^{-i}(1-x)^{-(r-i+1)}=(1-x)^{-(r+1)}$, we see that

$$
\sum_{e=0}^{k-r-1}\left(\begin{array}{c}
i+e-1 \\
e
\end{array}\right)\left(\begin{array}{l}
k-i-e-1 \\
k-r-1-e
\end{array}\right)=\left(\begin{array}{c}
k-1 \\
r
\end{array}\right)
$$


and by using the partial fraction decomposition

$$
F(x):=\sum_{e=0}^{k-r-1} \frac{(-1)^{e}}{e !(k-r-1-e) !} \cdot \frac{1}{x+e}=\frac{1}{x(x+1) \cdots(x+k-r-1)},
$$

we see that

$$
\begin{aligned}
& \sum_{e=0}^{k-r-1}\left(\begin{array}{c}
i+e-1 \\
e
\end{array}\right)\left(\begin{array}{c}
k-i-e-1 \\
k-r-1-e
\end{array}\right) \cdot(-1)^{i-1+e}\left(\begin{array}{c}
k+1 \\
i+e+1
\end{array}\right) \\
& =(-1)^{i-1} \frac{(k+1) !}{(i-1) !(r-i) !} \sum_{e=0}^{k-r-1} \frac{(-1)^{e}}{e !(k-r-1-e) !(i+e)(i+e+1)(k-i-e)} \\
& =(-1)^{i-1} \frac{(k+1) !}{(i-1) !(r-i) !}\left\{\frac{1}{k} F(i)-\frac{1}{k+1} F(i+1)+\frac{(-1)^{r-1}}{k(k+1)} F(r-i+1)\right\} \\
& =(-1)^{i-1}\left\{(k-r)\left(\begin{array}{c}
k \\
r-i
\end{array}\right)+\left(\begin{array}{c}
k-1 \\
r-i
\end{array}\right)+(-1)^{r-1}\left(\begin{array}{c}
k-1 \\
i-1
\end{array}\right)\right\} .
\end{aligned}
$$

Thus we have proved $S_{k, r, i}^{\star}=\frac{b_{k, r, i}}{2} \cdot \frac{B_{\boldsymbol{p}-k-1}}{k+1} \boldsymbol{p}$.

Let us take the sum $\sum_{\boldsymbol{k} \in I_{k, r, r+1-i}}$ of (16). Then we obtain

$$
\begin{aligned}
S_{k, r, r+1-i}^{\star}+\sum_{j=1}^{r-i}(-1)^{j} & \sum_{l=j}^{k-r+j-1} S_{l, j} S_{k-l, r-j, r+1-i-j}^{\star} \\
& +\sum_{j=r-i+1}^{r-1}(-1)^{j} \sum_{l=j+1}^{k-r+j} S_{l, j, j+i-r} S_{k-l, r-j}^{\star}+(-1)^{r} S_{k, r, i}=0 .
\end{aligned}
$$

We know that $S_{l, j} S_{k-l, r-j, r+1-i-j}^{\star}$ is a rational multiple of $B_{\boldsymbol{p}-l-1} B_{\boldsymbol{p}-k+l} \boldsymbol{p}$ for $1 \leq j \leq r-i$ and we also know that $S_{l, j, j+i-r} S_{k-l, r-j}^{\star}$ is a rational multiple of $B_{\boldsymbol{p}-l} B_{\boldsymbol{p}-k+l-1} \boldsymbol{p}$ for $r-i+1 \leq j \leq r-1$ by Theorem 2.4 and Proposition 4.6. Since $k$ is even, these are zero for every $l$. Therefore, we have

$$
S_{k, r, i}=(-1)^{r-1} \frac{b_{k, r, r+1-i}}{2} \cdot \frac{B_{\boldsymbol{p}-k-1}}{k+1} \boldsymbol{p}=(-1)^{r-1} \frac{a_{k, r, i}}{2} \cdot \frac{B_{\boldsymbol{p}-k-1}}{k+1} \boldsymbol{p} .
$$

\section{Sum formulas for $\mathcal{A}_{3}$-Finite multiple Zeta values}

For positive integers $k$ and $r$ such that $r \leq k$, we set

$$
T_{k, r}:=\sum_{\boldsymbol{k} \in I_{k, r}} \zeta_{\mathcal{A}_{3}}(\boldsymbol{k}), \quad T_{k, r}^{\star}:=\sum_{\boldsymbol{k} \in I_{k, r}} \zeta_{\mathcal{A}_{3}}^{\star}(\boldsymbol{k}) .
$$

From now on, we assume that $k$ is odd. We recall a formula

$$
\zeta_{\mathcal{A}_{3}}(k)=-\frac{k(k+1)}{2} \cdot \frac{B_{\boldsymbol{p}-k-2}}{k+2} \boldsymbol{p}^{2}
$$

proved by Sun [18, Theorem 5.1]. 
Theorem 5.1. Let $k$ and $r$ be positive integers satisfying $r \leq k$, and assume that $k$ is odd. Then we have

$$
(-1)^{r-1} T_{k, r}=T_{k, r}^{\star}=-\frac{k+1}{2}\left(\begin{array}{l}
k \\
r
\end{array}\right) \frac{B_{\boldsymbol{p}-k-2}}{k+2} \boldsymbol{p}^{2} .
$$

Proof. Since a congruence

$$
\begin{aligned}
& (-1)^{m-1}\left(\begin{array}{c}
p-1 \\
m
\end{array}\right) \\
& \equiv-1-\left(\sum_{m \leq n \leq p-1} \frac{1}{n}-\frac{1}{m}\right) p-\left(\sum_{m \leq n_{1} \leq n_{2} \leq p-1} \frac{1}{n_{1} n_{2}}-\frac{1}{m} \sum_{m \leq n \leq p-1} \frac{1}{n}\right) p^{2} \quad\left(\bmod p^{3}\right)
\end{aligned}
$$

holds for any odd prime $p$ and any positive integer $m$ with $m<p$ by [16, Lemma 4.1], one can deduce

$$
\begin{aligned}
& \sum_{j=0}^{k-r} \zeta_{\mathcal{A}_{3}}^{\star}\left(\{1\}^{k-r-j}\right) T_{j+r, r}^{\star} \\
& =\left(\begin{array}{l}
k \\
r
\end{array}\right)\left\{-\zeta_{\mathcal{A}_{3}}(k)-\zeta_{\mathcal{A}_{3}}^{\star}(k, 1) \boldsymbol{p}+\zeta_{\mathcal{A}_{3}}^{\star}(k+1) \boldsymbol{p}-\zeta_{\mathcal{A}_{3}}^{\star}(k, 1,1) \boldsymbol{p}^{2}+\zeta_{\mathcal{A}_{3}}^{\star}(k+1,1) \boldsymbol{p}^{2}\right\}
\end{aligned}
$$

from the identity (6) in the samy way as (18). Let us fix $0 \leq j<k-r$. By (14) and Proposition 4.6, if $j+r$ is odd, then $\zeta_{\mathcal{A}_{3}}^{\star}\left(\{1\}^{k-r-j}\right)$ is divisible by $\boldsymbol{p}$ and $T_{j+r, r}^{\star}$ is divisible by $\boldsymbol{p}^{2}$ and if $j+r$ is even, then $\zeta_{\mathcal{A}_{3}}^{\star}\left(\{1\}^{k-r-j}\right)$ is divisible by $\boldsymbol{p}^{2}$ and $T_{j+r, r}^{\star}$ is divisible by $\boldsymbol{p}$. Therefore, $\zeta_{\mathcal{A}_{3}}^{\star}\left(\{1\}^{k-r-j}\right) T_{j+r, r}^{\star}=0$ and we see that the left hand side of (23) is equal to $T_{k, r}^{\star}$. On the other hand, by using Proposition 4.1, Proposition 4.2 and (22), we see that the right hand side of (23) is equal to

$$
\begin{aligned}
\left(\begin{array}{l}
k \\
r
\end{array}\right) & {\left[\frac{k(k+1)}{2}-\frac{1}{2}\left\{-\left(\begin{array}{c}
k+2 \\
k
\end{array}\right)+k^{2}+3 k+1\right\}+(k+1)\right.} \\
& \left.-\frac{1}{2}\left\{-(k+2)+\left(\begin{array}{c}
k+2 \\
k
\end{array}\right)\right\}-(k+2)\right] \frac{B_{p-k-2}}{k+2} \boldsymbol{p}^{2} \\
= & -\frac{k+1}{2}\left(\begin{array}{c}
k \\
r
\end{array}\right) \frac{B_{\boldsymbol{p}-k-2}}{k+2} \boldsymbol{p}^{2} .
\end{aligned}
$$

Hence we have $T_{k, r}^{\star}=-\frac{k+1}{2}\left(\begin{array}{l}k \\ r\end{array}\right) \frac{B_{\boldsymbol{p}-k-2}}{k+2} \boldsymbol{p}^{2}$. By taking $\sum_{\boldsymbol{k} \in I_{k, r}}$ of (16)), we obtain

$$
T_{k, r}^{\star}+\sum_{j=1}^{r-1}(-1)^{j} \sum_{l=j}^{k-r+j} T_{l, j} T_{k-l, r-j}^{\star}+(-1)^{r} T_{k, r}=0 .
$$

Let us fix $1 \leq j \leq r-1$ and $j \leq l \leq k-r+j$. By Proposition 4.6, if $l$ is odd, then $T_{l, j}$ is divisible by $\boldsymbol{p}^{2}$ and $T_{k-l, r-j}^{\star}$ is divisible by $\boldsymbol{p}$ and if $l$ is even, then $T_{l, j}$ is divisible by $\boldsymbol{p}$ and $T_{k-l, r-j}^{\star}$ is divisible by $\boldsymbol{p}^{2}$. Therefore, we see that $T_{l, j} T_{k-l, r-j}^{\star}=0$ and this gives $(-1)^{r-1} T_{k, r}=T_{k, r}^{\star}$. 


\section{REFERENCES}

[1] S. Bang, J. E. Dawson, A. N. 't Woord, O. P. Lossers, V. Hernandez, Problems and solutions: solutions: a reciprocal summation identity: 10490, Amer. Math. Monthly 106 (1999), no. 6, 588590.

[2] D. M. Bradley, Multiple q-zeta values, J. Algebra 283 (2005), no. 2, 752-798.

[3] D. M. Bradley, Duality for finite multiple harmonic q-series, Disc. Math. 300 (2005), 44-56.

[4] K. Dilcher, Some q-series identities related to divisor functions, Disc. Math. 145 (1995), no. 1-3, 83-93.

[5] L. Euler, Demonstratio insignis theorematis numerici circa uncias potestatum binomialium, Nova Acta Acad. Sci. Petropol. 15, (1806), 33-43, reprinted in Opera Omnia, Ser. I, Vol. 16(2), B. G. Teubner, Leipzig, (1935), 104-116.

[6] A. Granville, A decomposition of Riemann's zeta-function, Lond. Math. Soc. Lecture Note Ser. 247, Cambridge, (1997), 95-101.

[7] M. Hirose, K. Imatomi, H. Murahara, S. Saito, Ohno type relations for classical and finite multiple zeta-star values, preprint, arXiv:1806.09299.

[8] M. Hoffman, Quasi-symmetric functions and mod $p$ multiple harmonic sums, Kyushu J. Math. 69 (2015), no. 2, 345-366.

[9] Y. Ohno, A generalization of the duality and sum formulas on the multiple zeta values, J. Number Theory 74 (1999), no. 1, 39-43.

[10] K. Oyama, Ohno's relation for finite multiple zeta values, to appear in Kyushu J. Math., arXiv:1506.00833.

[11] H. Prodinger, A q-analogue of a formula of Hernandez obtained by inverting a result of Dilcher, Australas. J. Combin. 21 (2000), 271-274.

[12] S. Roman, The harmonic logarithms and the binomial formula, J. Comb. Theory A 63 (1993) $143-163$.

[13] J. Rosen, Asymptotic relations for truncated multiple zeta values, J. Lond. Math. Soc. 91, (2015), no.2, $554-572$.

[14] S. Saito, N. Wakabayashi, Sum formula for finite multiple zeta values, J. Math. Soc. Japan 67 (2015), no. 3, 1069-1076.

[15] K. Sakugawa, S. Seki, On functional equations of finite multiple polylogarithms, J. Algebra 469 (2017), 323-357.

[16] S. Seki, The p-adic duality for the finite star-multiple polylogarithms, to appear in Tohoku Math. J., arXiv:1605.06739.

[17] S. Seki, S. Yamamoto, A new proof of the duality of multiple zeta values and its generalizations, preprint, arXiv:1806.04679.

[18] Z. H. Sun, Congruences concerning Bernoulli numbers and Bernoulli polynomials, Disc. Appl. Math. 105 (2000), no. 1-3, 193-223.

[19] L. Van Hamme, Advanced problem 6407, Amer. Math. Monthly 40, (1982), 703-704.

[20] J. Zhao, Wolstenholme type theorem for multiple harmonic sums, Int. J. Number Theory 4 (2008), no. $1,73-106$.

[21] J. Zhao, Finite multiple zeta values and finite Euler sums, preprint, arXiv:1507:04917.

[22] X. Zhou, T. Cai, A generalization of a curious congruence on harmonic sums, Proc. of Amer. Math. Soc. 135 (2007), no. 5, 1329-1333.

Mathematical Institute, Tohoku University, 6-3,Aoba, Aramaki, Aoba-Ku, Sendai 980-8578, JAPAN

E-mail address: shinichiro.seki.b3@tohoku.ac.jp 
Keio Institute of Pure and Applied Sciences (KiPAS), Graduate School of Science and Technology, Keio University, 3-14-1 Hiyoshi, Kohoku-ku, Yokohama, 223-8522, JAPAN

E-mail address: yamashu@math.keio.ac.jp 